\title{
Crooke Cell Adenoma
}

National Cancer Institute

\section{Source}

National Cancer Institute. Crooke Cell Adenoma. NCI Thesaurus. Code C154342.

A corticotroph adenoma composed of tumor cells with Crooke hyaline change. Ring-like cytokeratin expression is typical of these neoplasms. ACTH expression is dislocated to the cell periphery and juxtanuclear region. Ultrastructurally, intermediate filaments are arranged in a ring-like pattern. (WHO 2017) 